\title{
OUTCOMES OF OPEN THORACIC SURGICAL DECORTICATION IN PATIENTS WITH TUBERCULOUS EMPYEMA
}

Tahir Aslam, Amer Bilal, Manzoor Ahmed, Mohammad Abid, Abdul Baseer, Khurshid Ahmed Baloch*, Fawad Ali

Lady Reading Hospital MTI, Peshawar Pakistan, *Prince of Songkla University, Hat Yai, Songkhla, Thailand

\begin{abstract}
Objective: To analyze the outcome of open surgical decortication of tuberculous empyema patients at their late presentation.

Study Design: Cross sectional study.

Place and Duration of Study: Patients $(\mathrm{n}=128)$ with tuberculous empyema were subjected to decortication for one and half year starting from Jul 2017 to Dec 2018. Pre, intra, and post-operative characteristics of all patients were studied thoroughly with a 6-month follow up evaluation of the surgical outcomes.

Results: Preoperative conditions were as follows: $6.25 \%$ patient had Broncho pleural fistula, $90.9 \%$ patients were diagnosed with cough, 34.7 with night sweating, $64.2 \%$ chest pain and $79.6 \%$ chest tightness. Decortication was performed in all cases, with debridement of necrotic tissue and repair of air leaks in some patients. Seventy $(54.7 \%)$ patients went through right side decortication, whereas $56(43.7 \%)$ patients went through left sided decortication and $2(1.56 \%)$ patients had bilateral. Mortality was $2(1.56 \%), 1$ patient died of septicemia while 1 of pulmonary embolism. Whereas morbidity was $9(7 \%), 3$ patients had wound infection, 4 had incomplete lung expansion, 1 patient each had prolonged air leak and post-operative bleeding.

Conclusion: Delayed referral for surgery causes irreversible changes in the lung, and prolonged disease condition, which causes increased thickness of the pleura and injury to the underlying lung with a delayed recovery. Open decortication gives satisfactory results.
\end{abstract}

Keywords: Chylothorax, Decortication, Empyema necessitan, Tuberculous empyema.

This is an Open Access article distributed under the terms of the Creative Commons Attribution License (http://creativecommons.org/licenses/by/4.0), which permits unrestricted use, distribution, and reproduction in any medium, provided the original work is properly cited.

\section{INTRODUCTION}

Tuberculosis is the most common infectious disease in developing countries commonly occurring tuberculosis is pulmonary tuberculosis (PTB). However, it can effect several body parts. Tuberculosis other than PTB is known as extrapulmonary tuberculosis (EPTB). Pleural tuberculosis causing pleural effusion is one of the most common representation of EPTB ${ }^{1}$. Tuberculous empyema thoraces usually occur when caseous material from a superficial parenchymal cavity ruptures into the pleural space and when there is secondary Para tracheal lymph node involvement, in a direct extension of a paravertebral cold abscess, or hematogenous spread of infection ${ }^{2}$. Untreated or inadequately treated tuberculous empyema may result in Broncho pleural fistula, empyema necessitan, which often appear as

Correspondence: Dr Tahir Aslam, Trainee Thoracic Surgery, Lady Reading Hospital MTI, Peshawar Pakistan

Received: 10 Jan 2020; revised received: 29 Mar 2020; accepted: 30 Mar 2020 bronchopleurocutaneous fistula.

Pleural space is normally sterile but when itis contaminated with bacteria, it is converted to empyema. Three stages of empyema thoracic have so far been described by the American Thoracic Society; exudative stage, fibrinopurulent stage, and organizing stage ${ }^{3}$. In days 1-3 empyema thoracic is in exudative stage, which is a serious condition, occurs due to the increased permeability of the inflamed pleura. Whereas days 414 occurs fibrinopurulentstage, where an enhanced fibrin deposit leads towards empyema and loculations. The final stage occurring after day 14 with characteristics as follows: thickened pleura, resulting in an inelastic membrane condition known as 'the peel'which impedes lung movement known as trapped lung. Management of empyema depends on stage of disease at the time of presentation.

Tuberculous empyema causes more severe clinical outcomes than non-tuberculous etiologies 
such as accompanying fibrotic and cavitatory changes in the lung parenchyma, occurrence of Bronchopleural fistulae and poor general health of patients and also these patients may suffer from fibrothorax, intercostal space contraction and scoliosis due to chronicity of the disease ${ }^{4}$.

Anti-tuberculosis regimen, thoracentesis, and thoracic drainage are quite helpful in curing the early tuberculous empyema cases, whereas, chronic empyema patients mostly need to undergo a sequential course of treatment and surgery.

Various treatments have so far been employed to cure tuberculous empyema including antituberculous drugs, thoracentesis, tube thoracostomy, intrapleural fibrinolytic, video-assisted thoracoscopic surgery (VATS), open thoracotomy, space obliterating procedures and open window thoracostomy. However, every treatment has been reported with huge variations in their results 5,6 . This study was carried out to evaluate the effectiveness of open surgical intervention in managing tuberculous empyema thoraces in a large population of 128 patients.

\section{METHODOLOGY}

Data were collected from Jul 2017 to December 2018 in Thoracic surgery ward lady reading hospital Peshawar, followed by a 6-month proper follow-up process. One hundred twenty eight patients who were directly admitted or referred from other Pulmonology wards were included in the study. Patients who came directly to outpatient department were diagnosed with the help of history, examination and investigations which includes x-ray chest, Ultrasonography (USG), pleural fluid examination, closed pleural biopsy sputum AFB and gene expert. Computed tomography (CT) scan were done in most of cases to plan the surgery. All cases having tuberculous empyema thoraces or its complications were included in the study. Patients diagnosed with non-tuberculosis or other lung-associated diseases such as chylothorax, malignancy, and posttraumatic clotted haemothorax were excluded from the study. Post-operative pleura were sent for histopathological examination and pus was sent preoperatively for culture and sensitivity in all cases. Informed written consent was taken and the decortication procedures were well explained to the patients and their attendants.

Decortication was performed in all cases with multiloculated empyema in patients with trapped lung, broncho plural fistula, and empyema necessitan. In all these cases standard posterolateral thoracotomy incision was given with or without resection of rib. The pleural cavity was cleared from the debris of fibrinous tissue and pus. The thick encasing visceral pleural peel which was trapping the lung was carefully removed so that the lung expands on the table. The thick parietal pleura were removed from chest wall and costodiaphragmatic surface, taking care of the diaphragm and phrenic nerve. All significant air leaks were meticulously closed. Debridement of necrotic lung tissue was done and broncho pleural fistulas were closed. Chest tubes were inserted and the thoracotomy wound closed with vicryl, Intercostal block were given to all patients for post-operative pain control.

Once the decortication was performed, the drainage bottle was emptied by negative pressure. The color change in the chest drainage, breath sounds, and surgical incision were observed. Measures taken for the post-operative care of the patients were as follows: Daily chest radiographs were done to assess the expansion of lung and any collection. Low-pressure suction was employed. Patients were dealt with steam inhalation and incentive spirometry as early as possible along with nursing in an upright position. Adequate pain relief and antibiotics were administered intravenously till oral intake was resumed. Pus/debris were sent at the time of surgery for culture and sensitivity analysis, antibiotics were substituted depending on the culture report. Patients were evaluated postoperatively at 6 months' time.

SPSS software for Microsoft Windows (version 19.0, SPSS Inc., Chicago, IL) was employed for the statistical analysis. All the descriptive values were expressed as mean \pm standard devia- 
tion (SD) and median (range). Normal distribution of data was analyzed by one-way analysis of variance (ANOVA). A $p$-value of $<0.05$ was considered significant.

\section{RESULTS}

Out of 128 patients, $74(57.8 \%)$ were male and $54(42.2 \%)$ were female. Age range was from 10 years to 81 years with median age of 42 years. Most of these patients were already diagnosed and referred from other wardsor other hospitals (referred 112 patients 87.5\%). Patients admitted directly through our outpatient department were $16(12.5 \%)$. The common symptoms included cough $(90.9 \%)$, chest tightness $(79.6 \%)$, chest pain $(64.2 \%)$, fever $(37.6 \%)$, and night sweat $(34.7 \%)$

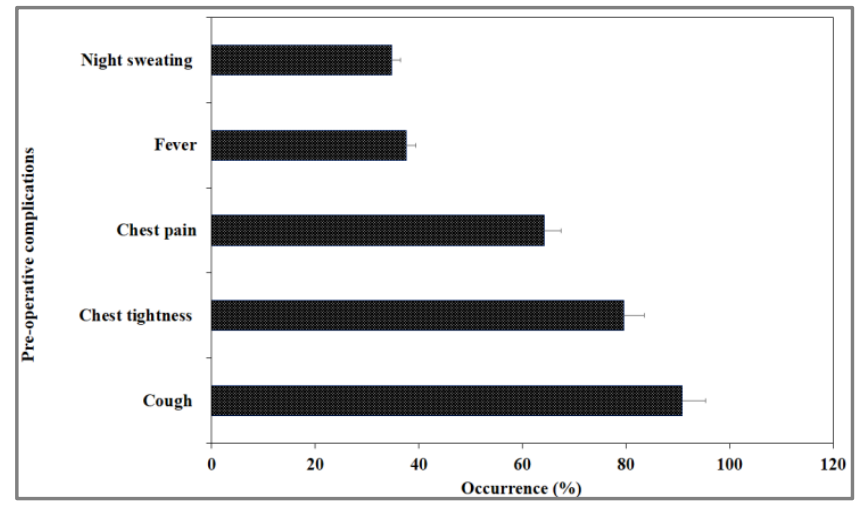

Figure-1: Pre-operative symptoms among the patients.

(fig-1). Seventy patients presented with right sided empyema, 54 patients presented with left sided empyema and two patients presented with bilateral empyema. Thirty two (25\%) patients amongst were in stage II, and 96 (75\%) were in stage III empyema. Some of these patients presented with complications of empyema, $1(0.785 \%)$ patient had empyema necessitain, $2(1.56 \%)$ patients had chest wall sinus and $8(6.25 \%)$ patients had broncho pleural fistula.

All patients were given general anesthesia with one lung ventilation, only one patient who was very moribund and not fit for general anesthesia was given local block for window formation.

Decortication was done in 98 (76.5\%) patients, $29(22.6 \%)$ patients were treated with thoracoplasty, $20(69 \%)$ of the patients treated with thoracoplasty went through partial thoracoplasty, whereas 9 (31\%) of them had complete thoracoplasty, moreover, one patient had clouget window formation. CT scan shows multiloculated pus, thickened pleura, and lung involvement (fig-2).

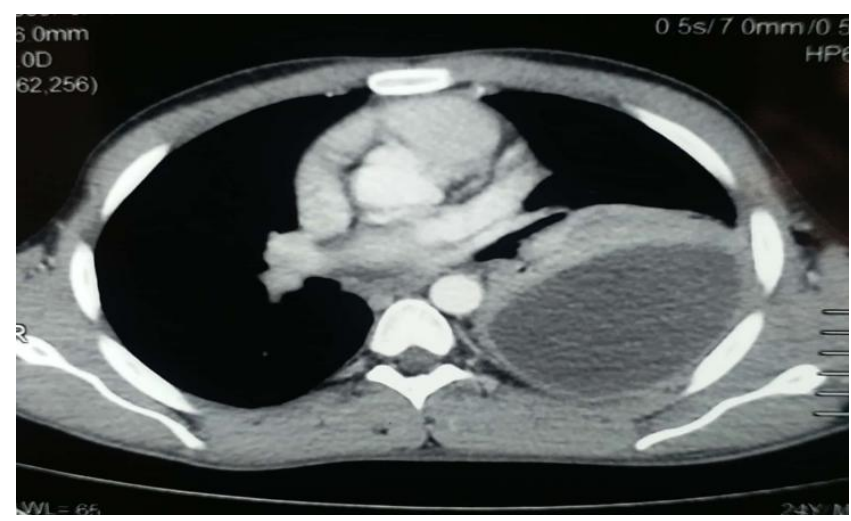

Figure-2: Pleural split sign with collection.

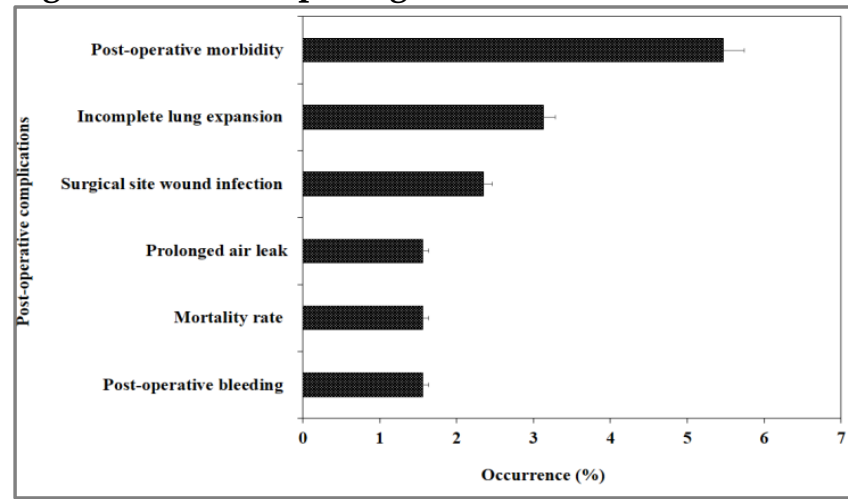

Figure-3: Post-operative complications among the patients.

All patients were extubated on the operation table, only one patient with 80 years age and comorbidities was on ventilator support and kept in ICU for three days after which he recovered.

Post-operative morbidity in our study was (7\%) that is 9 patients out of 128 patients. One patient developed post-operative bleeding, he was re-explored in emergency and hemostasis secured. Four patients had incomplete lung expansion they were encouraged with good nutrition aggressive physiotherapy and culture sensitive anti-biotic, three of these patient responded well to conservative therapy only one patient under- 
went partial thoracoplasty. One patient had prolonged air leak, which also responded to conservative therapy. Surgical site wound infection developed in 3 patients pus culture was taken in these cases and treated according to culture sensitive anti-biotic therapy (fig-3).

Two patients passed away in this study with the mortality rateof $1.56 \%$. One patient developed pulmonary embolism and one patient developed septicemia and did not recover. A six-month follow up was scheduled with all the patients to evaluate post-operative complications.

\section{DISCUSSION}

Most of the early tuberculous empyema cases have reportedly been successfully administered with a range of anti-tuberculosis regimen, thoracentesis, and thoracic drainage ${ }^{8,9}$. While, patients with chronic empyema and with complications of tuberculous empyemarequire a longand sequential course of treatment and surgery ${ }^{10,11}$. Tuberculous empyema generally brings worse outcomes than in non-tuberculous etiologies because of the associated complications such as fibrocavitatory parenchymal disease, frequent bronchopleural fistulae ${ }^{19}$.

Our study shows that open decortication is the first line therapy in advanced post tuberculous pleural empyema as reported by Ahmed et al (2016) ${ }^{18}$. As most of the patients in our case were shifted from other unites and before visiting us, for more than two weeks, they were treated with chest intubation butdid not respond to the treatment. After decortication, these patients were discharged home within a week. Same results as reported by Deewan et al (2010)12 were observed, surgical intervention in tuberculous empyema could reduce the hospital stay of the patients. Even though post-operative complications such as air leak ${ }^{19,20}$, positive Intraoperative cultures $^{20}$ are concomitant, yet, surgical decortication is considered as the first line treatment ${ }^{21}$.

Controversies exist about the management of early empyema in developed countries because of lacking clear-cut guidelines to follow ${ }^{13}$. With the introduction of Video Assisted Thoracoscopic Surgery (VATS), many studies have shown that patients have now bypassed medical thoracostomy and proceed directly to VATS4. Although the role of VATS in stage III empyema is very much debatable ${ }^{14}$.

It is important to remember that not only multiloculation but also thick debris resembling cheese will give a dry yield. Sometimes, after an initial drainage of pus, the only content is air, which remains because the lung is unable to expand, because of the thick unyielding visceral pleura. It is that the true nature of the pleural collection and the status of the lung can be determined only by a CT scan ${ }^{15,16}$. Most centers in the devolping countries still manage complications of pneumonia with chest radiographs alone or at the most with an ultrasonography. The world is gradually giving importance to other modalities of imaging for timely detection and intervention of pleural space infections ${ }^{17}$.

The results from this studies were quiet similar with Chen et al (2015), Kumar et al (2017) and Shin et al (2013) who also reported permanent air leakage, positive intraoperative cultures, and mortality during decortication and also found that decortication is the more suitable way to cure tuberculous empyema. Moreover, during open thoracotomy in our study, three significant changes effected morbidity are as follows: (a) consolidation, (b) cavitary necrosis and (c) poor compliance/fibrosis with poor expansion on Ambu bag ventilation.

This study has shown that delayed referral increases morbidity by not only increasing parenchymal involvement but also causing a state of malnutrition. The effect on the lung by the inflammatory process and the compression by the thickened pleura that has stopped physiologically functioning has a variable effect. Apart from necrosis and consolidation, which may be similar in patients presenting before and after 3 weeks, the incidence of reduced compliance of the lung and loss of lung tissue in chronic cases has a major effect on postoperative recovery. 


\section{CONFLICT OF INTEREST}

This study has no conflict of interest to be declared by any author.

\section{REFERENCES}

1. Lazarus AA, McKay S, Gilbert R. Pleural tuberculosis. Dis Mon 2007; 53(1): 16-21.

2. Sahn SA, Iseman MD. Tuberculous empyema. Semin Respir Infect 1999; 14(1): 82-87.

3. Molnar TF. Current surgical treatment of thoracic empyema in adults. Eur J Cardiothorac Surg 2007; 32(1): 422-30.

4. Luh SP, Chou MC, Wang LS, Chen JY, Tsai TP. Video-assisted thoracoscopic surgery in the treatment of complicated parapneumonic effusions or empyemas: outcome of 234 patients. Chest 2005; 127(1): 1427-32.

5. Cardillo G, Carleo F, Carbone L, Martino MD, Salvadori L, Petrella L, et al. Chronic postpneumonic pleural empyema: comparative merits of thoracoscopic versus open decortication. Eur J Cardiothorac Surg 2009; 36(5): 914-18.

6. American Thoracic Society Management of non-tuberculous empyema. Ann Rev Respir Dis 1962; 85(1): 935-36.

7. Kundu S, Mitra S, Mukherjee S, Das S. Adult thoracic empyema: A comparative analysis of tuberculous and nontuberculous etiology in 75 patients. Lung India 2010; 27(4): 196-201.

8. Bailey KA, Bass J, Rubin S, Borrowman N. Empyema management: twelve years' experience since the introduction of videoassisted thoracoscopic surgery. J Laparoendosc Adv Surg Tech 2005; 15(3): 338-41.

9. Casali C, StorelliES, Di Prima E, Morandi U. Long-term functional results after surgical treatment of parapneumonic thoracic empyema. Interact Cardiovasc Thorac Surg 2009; 9(1): 74-78.

10. Shahin Y, Duffy J, Beggs D, Black E, Majewski A. Surgical management of primary empyema of the pleural cavity: outcome of 81 patients. Interact Cardiovasc Thorac Surg 2010; 10(4): 565-67.
11. Carballo M, Maish MS, Jaroszewski DE, Holmes CE. Videoassisted thoracic surgery (VATS) as a safe alternative for the resection of pulmonary metastases: a retrospective cohort study. J Cardiothorac Surg 2009; 4(1): 13-19.

12. Deewan RK. Surgery for pulmonary tuberculosis - a 15-year experience. Eur J Cardiothorac Surg 2010; 37(1): 473-77.

13. Sonmezoglu Y, Turna A, Cevik A, Demir A, Sayar A, Dincer Y, et al. Factors affecting morbidity in chronic tuberculous empyema. Thorac Cardiovasc Surg 2008; 56(2): 99-102.

14. Scarci M, Zahid I, Billé A, Routledge T. Is video-assisted thoracoscopic surgery the best treatment for paediatric pleural empyema? Interact Cardiovasc Thorac Surg 2011; 13(1): 70-76.

15. Tacconi F, Pompeo E, Fabbi E, Mineo TC. Awake video-assisted pleural decortication for empyema thoracis. Eur J Cardiothorac Surg 2010; 37(3): 594-601.

16. Barbetakis N, Paliouras. eComment: the role of video-assisted thoracoscopic surgery in the management of tuberculous empyemas. Interact Cardiovasc Thorac Surg 2009; 8(1): 337-38.

17. Kerti CA, Miron I, Cozma GV, Burlacu ON, Tunea CP. The role of surgery in the management of pleuropulmonary tuberculosis -seven years' experience at a single institution. Interact Cardiovasc Thorac Surg 2009; 8(3): 334-37.

18. Ahmed S, Azam H, Basheer I. Is open decortication superior to fibrinolytic therapy as a first line treatment in the management of pleural empyema?. Pak J Med Sci 2016; 32(2): 329.

19. Chen B, Zhang J, Ye Z, Ye M, Ma D, Wang C, et al. Outcomes of video-assisted thoracic surgical decortication in 274 patients with tuberculous empyema. Ann Thorac Cardiovascular Surg 2015: oa-14.

20. Kumar A, Asaf BB, Lingaraju VC, Yendamuri S, Pulle MV, Sood J. Thoracoscopic decortication of stage III tuberculous empyema is effective and safe in selected cases. Ann Thoracic Surg 2017; 104(5): 1688-94.

21. Shin JA, Chang YS, Kim TH, Haam SJ, Kim HJ, Ahn CM, et al. Surgical decortication as the first-line treatment for pleural empyema. J Thoracic Cardiovas Surg 2013; 145(4): 933-39. 